\title{
Cartilage Repair in the Knee: Part II
}

\author{
Jack Farr, M.D. ${ }^{2}$ James L. Cook, DVM, Ph.D. ${ }^{1}$ \\ ${ }^{1}$ William and Kathryn Allen Distinguished Professor in Orthopaedic \\ Surgery; Director, Comparative Orthopaedic Laboratory; University \\ of Missouri, Columbia, Missouri \\ 2 Medical Director, Cartilage Restoration Center of Indiana; Director, \\ Ortholndy Sports Medicine Fellowship, Indiana Orthopaedic \\ Hospital, South, Greenwood, Indiana
}

J Knee Surg 2012;25:1-2.

This represents Part II of a comprehensive overview of the current state of the art of knee cartilage repair. We hope you enjoyed Part I that laid a firm foundation for assessing the patient's knee prior to surgery.

In this issue, we are fortunate to have authors who are not only familiar with the current treatments available in the United States, but also are exploring new treatment approaches. Fortier will establish the scientific basis on marrow stimulation, which sets the stage for Gomoll's article on current clinical applications and then the potential augmentation options that may improve marrow stimulation
Address for correspondence and reprint requests James L. Cook, DVM, Ph.D., William and Kathryn Allen Distinguished Professor in Orthopaedic Surgery, University of Missouri, 900 East Campus Drive, Columbia, MO 65211 (e-mail: CookJL@missouri.edu).

outcomes and durability. Autologous chondrocyte implantation (ACI) was one of the major factors in jump starting the clinical field of cartilage repair and restoration. Kon begins with that milestone and brings us up to date on the subsequent generations of $\mathrm{ACl}-$ now available in Europe. Farr introduces the topic of particulated allograft cartilage, which is in its infancy in clinical applications; and finally, Cole explores nontraditional modifications of articular cartilage.

We hope you are enjoying our continued journey in knee cartilage repair. We will continue the saga in Part III.
DOI http://dx.doi.org/ 10.1055/s-0032-1309289. ISSN 1538-8506. 